\title{
Foot ulcer simulation training (FUST): are podiatrists FUST with long-term clinical confidence?
}

\author{
Peter A Lazzarini ${ }^{1,2,3^{*}}$, Vanessa $\mathrm{Ng}^{1,2}$, Patricia M Régo ${ }^{4,5}$, Suzanne S Kuys ${ }^{1,6}$, Scott Jen ${ }^{7}$ \\ From Australasian Podiatry Council Conference 2013 \\ Sydney, Australia. 2-5 June 2013
}

\section{Background}

Foot ulcers are a leading cause of diabetes-related hospitalisations. Clinical training has been shown to be beneficial in foot ulcer management. Recently, improved selfconfidence in podiatrists was reported immediately after foot ulcer simulation training (FUST) pilot programs. This study aimed to investigate the longer-term impacts of the FUST program on podiatrists' self-confidence over 12 months in a larger sample.

\section{Methods}

Participants were podiatrists attending a two-day FUST course comprising web-based interactive learning, lowfidelity part-tasks and high-fidelity full clinical scenarios. Primary outcome measures included participants' selfconfidence measured pre-, (immediately) post-, 6-month post- and 12-month post-course via a purpose designed 21 -item survey using a five-point Likert scale $(1=$ Very limited, $5=$ Highly confident). Participants' perceptions of knowledge gained, satisfaction, relevance and fidelity were also investigated. ANOVA and post hoc tests were used to test any differences between groups.

\section{Results}

Thirty-four participants completed FUST. Survey response rates were $100 \%$ (pre), $82 \%$ (post), $74 \%$ (6-month post), and $47 \%$ (12-month post). Overall mean scores were 3.13 (pre), 4.49 (post), 4.35 (6-month post) and 4.30 (12-month post) $(p<0.05)$; post hoc tests indicated no differences between the immediately, 6-month and 12-month post group scores $(p>0.05)$. Satisfaction, knowledge, relevance and fidelity were all rated highly.

\section{Conclusion}

This study suggests that significant short-term improvements in self-confidence to manage foot ulcers via simulation training are retained over the longer term. It is likely that improved self-confidence leads to improved foot ulcer clinical practice and outcomes; although this requires further research.

\section{Author details}

${ }^{1}$ Allied Health Research Collaborative, Metro North Hospital \& Health Service, Queensland Health, Brisbane, Queensland, 4032, Australia. ${ }^{2}$ Department of Podiatry, Metro North Hospital \& Health Service, Queensland Health, Brisbane, Queensland, 4032, Australia. ${ }^{3}$ School of Clinical Sciences, Queensland University of Technology, Brisbane, Queensland, 4059, Australia. ${ }^{4}$ School of Medicine, The University of Queensland, Brisbane, Queensland, 4072, Australia. ${ }^{5}$ Clinical Skills Development Service, Centre for Healthcare Improvement, Queensland Health, Brisbane, Queensland, 4029, Australia. ${ }^{6}$ Musculoskeletal Research Program, Griffith Health Institute, Griffith University, Gold Coast, Queensland, 4222, Australia. DDepartment of Podiatry, West Moreton Hospital \& Health Service, Queensland Health, Ipswich, Queensland, 4305, Australia.

Published: 31 May 2013

doi:10.1186/1757-1146-6-S1-022

Cite this article as: Lazzarini et al.: Foot ulcer simulation training (FUST): are podiatrists FUST with long-term clinical confidence? Journal of Foot and Ankle Research 2013 6(Suppl 1):O22.

\footnotetext{
* Correspondence: Peter_Lazzarini@health.qld.gov.au

${ }^{1}$ Allied Health Research Collaborative, Metro North Hospital \& Health Service,

Queensland Health, Brisbane, Queensland, 4032, Australia
}

Full list of author information is available at the end of the article 